
\section{$C_{\text {chitsis }}$}

${ }^{1}$ Department of Paediatrics, JIPMER, Puducherry, India ${ }^{2}$ Department of Pathology, JIPMER, Puducherry, India ${ }^{3}$ Department of Medical Oncology, JIPMER, Puducherry, India

\section{Correspondence to} Dr Venkatesh Chandrasekaran, Department of Paediatrics, JIPMER, Dhanvantri Nagar, Puducherry 605006, India; cvenkatesh@hotmail.com

Received 5 August 2015 Revised 13 December 2015 Accepted 3 January 2016 Published Online First 25 January 2016

\title{
Persistent tachypnoea in an infant with cystic lung lucencies on CT scan
}

\author{
Vijayasekharan Kalasekhar, ${ }^{1}$ Selvam Nithiya, ${ }^{1}$ Venkatesh Chandrasekaran, ${ }^{1}$ \\ Reena Gulati, ${ }^{1}$ Sajini Elizabeth Jacob, ${ }^{2}$ Biswajit Dubashi ${ }^{3}$
}

A 1 year and 8 months old boy, with mild motor developmental delay and right hemiparesis following a perinatal brain injury, was brought with a

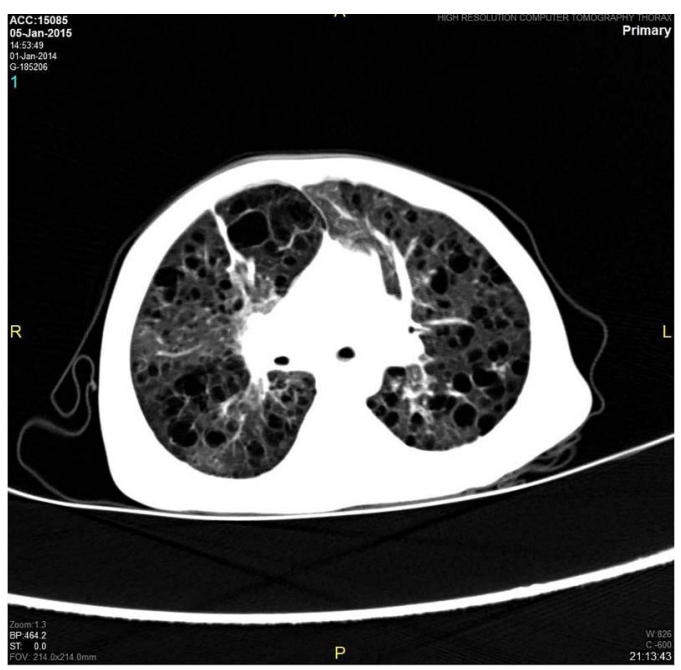

Figure 1 High-resolution CT scan of the thorax showing multiple cystic lesions of irregular size involving the entire lung fields. history of fast breathing and cough for 2 months and low-grade fever of 10 days duration. He was second born to non-consanguineously wed parents by spontaneous vaginal delivery with delayed cry at birth and neonatal intensive care unit stay for 10 days. There was no exposure to cigarette smoke. On examination there was mild central cyanosis with pan digital clubbing; pulse rate was 102 per minute; respiratory rate was 44 per minute; oxygen saturation of $89 \%$ which improved to $95 \%$ with $2 \mathrm{~L} / \mathrm{min}$ of oxygen. On systemic examination, cardiovascular status was found to be normal; respiratory examination revealed coarse crepitations bilaterally in the entire lung fields; abdominal examination revealed normal sized liver and a palpable spleen tip; neurological examination revealed mild right hemiparesis. His investigations revealed normal blood counts with mild microcytic hypochromic anaemia; sterile blood culture; chest radiograph revealed bilateral streaky interstitial opacities with honey comb pattern; work-up for TB and immunodeficiency states returned negative results. $\mathrm{He}$ was treated with ceftriaxone injection for 7 days without any improvement. A high-resolution CT scan of the chest revealed multiple cystic lesions of irregular size involving the parenchyma of entire
To cite: Kalasekhar $V$, Nithiya S, Chandrasekaran V, et al. Thorax 2016;71: 575-576.

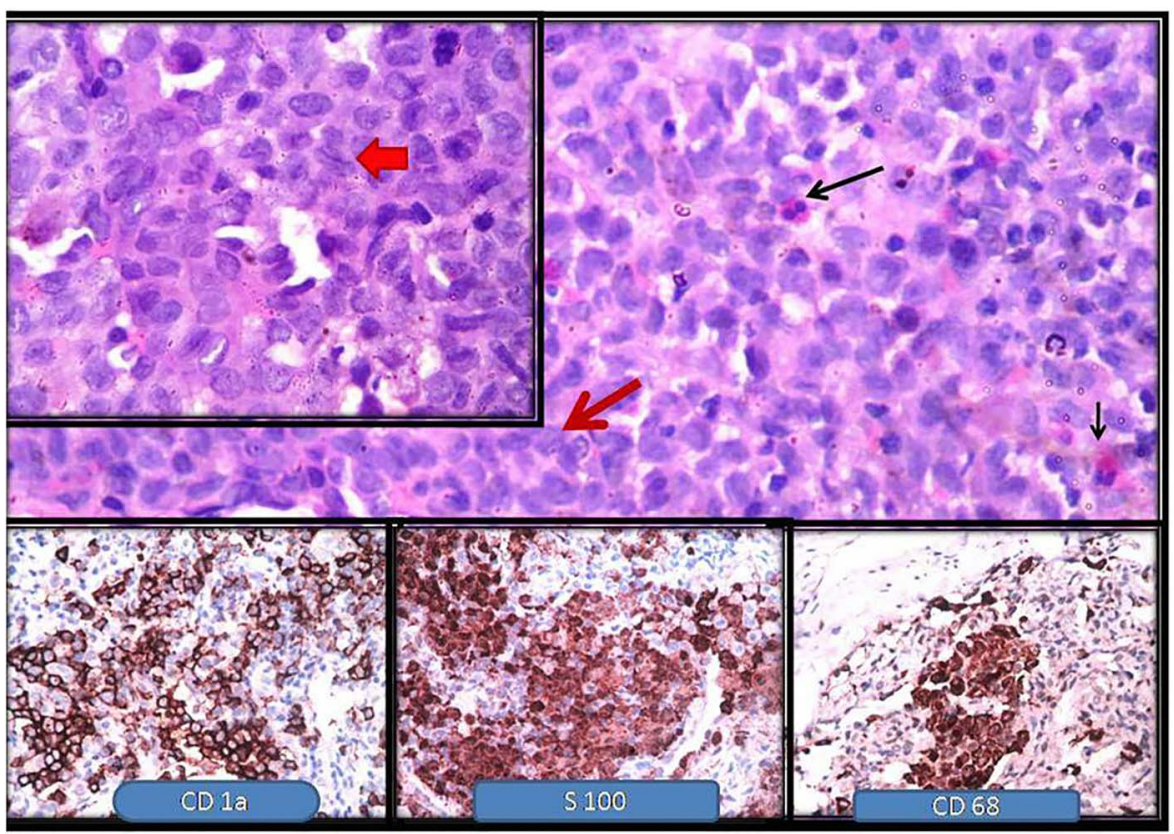

Figure 2 The cellular infiltrate composed of Langerhans cells (red arrow) admixed with eosinophils (black arrow) (H\&E $\times 100)$. Left upper inset-Langerhans cells with convoluted grooved nuclei (red arrow $\times 1000)$. Lower insetLangerhans cells showing CD1a, S100 and CD68 positivity (DAB $\times 400)$. 


\section{Chest clinic}

lung fields (figure 1). Lung biopsy revealed nodular infiltrates of Langerhans cells, alveolar macrophages with immunohistochemical staining positive for CD1a, CD68, S100 (figure 2). Bone marrow examination, liver biopsy, bone scan and echocardiogram were normal. MRI of the brain was uninformative except for gliosis in left frontoparietal lobe. He was managed as isolated pulmonary langerhan cell histiocytosis (PLCH) with pulmonary dysfunction and treated with 6 weeks course of oral prednisolone and weekly vinblastine therapy. On follow-up his tachypnoea had decreased gradually and considerably with improved effort tolerance.

Langerhans cell histiocytosis (LCH) is a rare multisystem disease of unknown aetiology characterised by clonal proliferation and accumulation of dendritic cells in many organ systems resulting in a heterogenous presentation. ${ }^{1}$ Lung involvement is a well-known complication of multisystem $\mathrm{LCH}$, both in children and in adults. Isolated PLCH, on the other hand, has been reported largely in adult smokers, but very rarely in children. ${ }^{2}$ The classical radiological changes described in pulmonary LCH include bilateral nodular and reticulonodular infiltrates predominantly involving the upper and mid-lung zones (in adults) in the early stages and becoming predominantly cystic at later stages. ${ }^{3}$ In children, the subpleural parenchyma and costophrenic recess are more often involved than in adults. ${ }^{3}$ The pathological changes in PLCH consist of granuloma (rich in Langerhans cells, eosinophils, lymphocytes and macrophages) formation and peribronchiolectasia. Intervening lung parenchyma may appear normal. ${ }^{1}$ Langerhans cells can be identified based on the presence of an eosinophilic cytoplasm, elongated nuclei which have clefts and folds. Immunohistochemical staining will identify S100, CD1a antigen and CD207 (Langerin) which are expressed by histiocytes. For patients with progressive decline in lung function, corticosteroid therapy is indicated. If unresponsive, treatment with vinblastine or methotrexate is initiated.

Acknowledgements We thank the Departments of Radiodiagnosis and Paediatric Surgery, JIPMER for helping us with radiological diagnosis and lung biopsy, respectively. Our special thanks to Dr Srinivas, Consultant, Pulmonary Medicine, JIPMER, for his inputs regarding the differential diagnosis.

Contributors All authors were involved in disease management, literature review, drafting of manuscript and critically reviewing it for intellectual content.

Competing interests None declared.

Patient consent Obtained.

Provenance and peer review Not commissioned; externally peer reviewed.

\section{REFERENCES}

1 Ng-Cheng-Hin B, O'Hanlon-Brown C, Alifrangis C, et al. Langerhans cell histiocytosis: old disease new treatment. QJM 2011;104:89-96.

2 Ha SY, Helms P, Fletcher M, et al. Lung involvement in Langerhans' cell histiocytosis: prevalence, clinical features and outcome. Pediatrics 1992;87:446-69.

3 Seely JM, Salahudeen S Sr, Cadaval-Goncalves AT, et al. Pulmonary Langerhans cell histiocytosis: a comparative study of computed tomography in children and adults. J Thorac Imaging 2012;27:65-70. 